\title{
Chiara Licameli \\ Voci di donne per una Italia Unita: «La donna Italiana: giornale politico-letterario»
}

\begin{abstract}
Il presente contributo propone un'analisi de «La donna italiana: giornale politico-letterario», periodico pubblicato a Roma nel 1848 ad opera di Cesare Bordiga. Il giornale - edito per soli 24 numeri, dal 22 aprile all'11 novembre dello stesso anno - nasce con lo scopo di sottolineare l'importanza delle donne nei moti risorgimentali in atto e si muove su una doppia linea, quella politica e quella letteraria: accanto alla cronaca dei rivolgimenti sono presenti poesie, racconti, biografie e aneddoti volti a sollecitare le cittadine e i cittadini a contribuire alla lotta per l'Unità. Tra le pagine del periodico si ritrovano le voci delle poetesse e patriote più famose del primo Ottocento italiano affiancate a quelle di personalità di rilievo come Filippo Meucci e Mazzini. I pensieri e le vite delle donne che collaborano al giornale formano così un catalogo parlante delle donne illustri viventi in un'epoca in cui i cataloghi biografici hanno una specifica funzione civile e formativa. La rivista mette in evidenza, dunque, come l'esaltazione della donna in questa fase della storia unitaria sia un modo per contribuire a costruire l'Italia e contiene diversi elementi di indagine sul contesto culturale della Roma risorgimentale, nonché sul ruolo dello Stato Pontificio nel processo unitario. I compilatori, infatti, sottolineano più volte la parte attiva che Roma ha nei moti e il fatto che la città debba essere considerata la guida simbolica della nazione in quanto rappresentante della gloria passata della Roma Imperiale.
\end{abstract}

\section{1. «La donna italiana» nella Roma risorgimentale}

Il 12 marzo 1847 un editto autorizza la stampa periodica dello Stato Pontificio a occuparsi di «argomenti di amministrazione e di storia contemporanea» ${ }^{1}$ per la prima volta dopo la Restaurazione. L'approvazione della normativa modifica un tessuto editoriale romano in cui vanno per la maggiore riviste culturali quali il «Giornale arcadico», «L'Album» e lo «Spigolatore» ${ }^{2}$ e dà più ampio spazio ai fogli politici. L'allentamento della censura provoca il fiorire e l'affiancarsi di fogli dalla prospettiva giobertiana come «L'Epoca», appoggiato da Mamiani, a giornali di orientamento liberale radicale, come il «Contemporaneo», diretto da Sterbini. ${ }^{3}$ In questo quadro, il 22 aprile del 1848, si inserisce «La donna italiana: giornale politico-letterario» che nasce con l'obiettivo del suo direttore, Cesare Bordiga, 4 di fondare una rivista che si occupi al tempo stesso di politica e di cultura. Bordiga vuole sottolineare l’importanza della partecipazione femminile alle rivolte in atto

The e-journal «altrelettere» is hosted at the URL: http://www.altrelettere.uzh.ch, in accordance with the Open Access Policy of the University of Zurich. Please cite this article as follows: Chiara LICAMELI, Voci di donne per una Italia Unita: «La donna Italiana: giornale politico-letterario», , in «altrelettere», 16.3.2018, DOI: 10.5903/al_uzh-37.

(C) This article is licensed under a Creative Commons Attribution 2.5. Switzerland (CC BY-NC-ND 2.5). Please read the license terms on the website: http://creativecommons.org/licenses/by-nc-nd/2.5/ch/deed.en 
in Italia e si muove su una doppia linea: l'esaltazione della donna e l'esaltazione della patria. I periodici rivolti ad un pubblico femminile dal ' 46 in poi sono diffusi nella Penisola e consistono principalmente in riviste di moda o in giornali pedagogici: basti pensare a «Il Folletto», che accoglie biografie di donne celebri, o a «Il Messaggero delle Dame», pensato per l'educazione morale e civile della donna (SOLDANI 2004, 309; MORI 2011, 71-73). «La donna italiana» si configura in questo contesto come un giornale politico-pedagogico 5 la cui specificità è quella di dare predominanza al tema patriottico, ${ }^{6}$ rivolgendosi non solo alle donne, ma anche agli uomini. Dal ' 48 , del resto, si registra in Italia una nuova partecipazione femminile agli eventi politici in atto: le donne intervengono nei moti risorgimentali con prose e versi patrii, rintracciabili su periodici, raccolte collettive, antologie, scritti accademici (CHEMELLO 2010 e 2014), ma anche come combattenti, infermiere, giornaliste, e costituiscono una rete coesa e attiva (MORI 2011, Bertolo 2011, Doni et al. 2011, Chemello 2014).

La rivista (pubblicata per soli 24 numeri e soppressa l'11 novembre dello stesso anno), viene data alle stampe nel corso di mesi fondamentali per la storia dello Stato Pontificio. Nei giorni in cui nasce il foglio è alle porte la prima guerra di Indipendenza, e Pio IX è schierato contro l'Impero austriaco, ma appena una settimana dopo, il 29 aprile, la sua posizione cambia. Il Pontefice pronuncia in un Concistoro una Allocuzione ${ }^{7}$ in cui non solo prende una posizione neutrale nei confronti dell'Austria, ma rifiuta la possibilità di essere coinvolto nei moti e di porsi a capo di un governo federale. Il popolo, animato da Ciceuracchio, ${ }^{8}$ si oppone fortemente alla decisione del Pontefice e manifesta segni di insofferenza sempre maggiori che si traducono in violenti tumulti. Nel tentativo di riguadagnare il favore delle frange più moderate Pio IX nel mese di settembre nomina Pellegrino Rossi Primo Ministro, 9 ma il 15 novembre questo viene assassinato e scoppiano nuove e più violente sommosse. Pio IX è costretto a fuggire a Gaeta e il 9 febbraio viene proclamata la Repubblica - guidata dai triumviri Armellini, Mazzini e Saffi - che avrà corso fino al 4 luglio di quello stesso anno, quando il papa, grazie all'aiuto francese, riuscirà a riguadagnare il potere. «La donna italiana» interrompe la pubblicazione pochi giorni prima di 
questo evento e racconta da una prospettiva interna i mesi che precedono la fuga del pontefice; la rivista dà ampio spazio alla cronaca degli atti compiuti dalle donne in favore dell'Unità1o e accoglie le voci di patriote provenienti da tutta Italia.

\section{Il Programma, i protagonisti, i temi affrontati}

Il Programma alle donne d'Italia, pubblicato da Bordiga in apertura de «La donna italiana» rende esplicito sin da subito lo scopo principale del giornale, ossia quello di formare una nuova classe di giovani donne colte e impegnate politicamente:

Un funesto errore dominò le menti dei padri, quello cioè che ad altro la donna non dovesse intendere, tranne alle domestiche cure. Questo pregiudizio si alimentò finche l'amor patrio venne apposto a delitto, finché il proferire la parola Italia fruttava persecuzioni e catene [...] Ma d'improvviso i nostri fati cangiarono [...]. Iddio mosso a pietà delle nostre sventure, avea mandato in terra uno dei suoi angeli a recarvi consolazione e quell'angelo era Pio IX. [...] Il bisogno di educare la donna venne universalmente sentito, si deplorò quell'errore che le aveva tolto l'antica energia rendendola un essere debole. [...] Si! - Codardamente vi calunnia, o donne d'Italia, chi osa affermare che più non possiate nutrire quei sentimenti e quegli affetti che un giorno vi resero degne di cotanta ammirazione. Voi avete e anima e cuore, voi nate in questa terra divina, non potete e non dovete ignorare che in ogni zolla di questa racchiude le ceneri di un'eroe [sic], che le istorie di ogni italiana città, se hanno molte e molte altre che narrano mille gesta sublimi dei nostri padri, che resero l'Italia più grande di ogni altra straniera nazione. Alle commosse popolazioni voi lo attestate o magnanime donne siciliane, voi o lombarde, coi prodigi della carità cittadina, quando sfidaste i perigli e la morte in difesa di una causa santissima; incoraggiando i vostri cari alla pugna, combattendo al loro fianco, ed un sovrumano eroismo infondeste nel petto dei figli, dei consorti, dei padri, allorché sorsero uniti in un patto ad abbattere l'aborrita tirannide. Voi pure lo attestate o generose liguri donne che bronzi guerreschi c'inviaste per fulminare gli odiati oppressori, e voi tutte insomma che prodigaste oro [...]. Tante virtù che destarono ammirazione profonda nei petti generosi, ci hanno incoraggiato a dare alla luce un Giornale che alla donna dirigesse libere parole, alimentandone il coraggio, dando contezza di ogni opra da essa intrapresa in vantaggio della patria, e ispirando nella sua anima forti virtù (BoRDIGA, LDI, n.1, 1). ${ }^{11}$

Bordiga in primo luogo identifica Pio IX come la guida che porterà l'Italia all'Unità, in seconda istanza sottolinea il bisogno di curare l'istruzione della donna, infine, riallacciandosi al discorso foscoliano, evidenzia la necessità di risollevare le sorti di una nazione che ha dato i natali a personaggi illustri. Gli 
scopi del giornale sono fin da subito evidenti: l'esaltazione di Pio IX e della patria, l'istruzione della donna e la sua formazione di patriota e cittadina, l'esortazione alla donazione di denaro per la causa pubblica. La presenza femminile tra le pagine del periodico è abbondante e variegata: accanto ai nomi di autrici romane come Teresa Gnoli e Elena Montecchi Torti, ${ }^{12}$ figurano quelli di Francesca Cantalamessa (Ascoli), Elvira Giampieri Rossi (Firenze), Caterina Franceschi Ferrucci (Narni), Assunta Pieralli (Perugia), Carlotta Gommi (Forlì), Luisa Amalia Paladini (Lucca), Maria Giuseppa Guacci Nobile (Napoli), ma anche di altre italiane meno note come Amelia Calani Sarteschi Carletti, Elvira Giampieri Rossi e Isabella Rossi Gabardi Brocchi (tutte fiorentine); ${ }^{13}$ tra i collaboratori ci sono invece personalità di rilievo come Filippo Meucci. ${ }^{14}$ I contenuti sono diversificati: nei ventiquattro numeri sono presenti articoli dedicati alla cronaca degli ultimi avvenimenti di guerra e all'educazione femminile, sei biografie di donne illustri15 e rassegne teatrali. Sono presenti inoltre quattro racconti a puntate ${ }^{16}$ e ventidue poesie di carattere patriottico, ${ }^{17}$ nonché uno spazio dedicato alle Varietà in cui sono raccolti articoli di varia natura: si passa da Poche parole di una donna in difesa della propria causa (LDI, n.1, 4) a Pensieri e consigli di celebri donne (LDI, n.20, 79) ${ }^{18}$ in cui sono riportati alcuni aforismi di Nonon de Lenclos, Anne Marguerite Dunoyer, Marie-Thérèse Rodet Goeffrin, Julie de Lespinasse e Madame Neher riguardanti l'amore e la vanità.

L'educazione è il tema principale del giornale, ${ }^{19}$ in cui è ribadita più volte l'affermazione «educhiamo gli intelletti e diventeremo liberi» (CARLETTI, LDI, $\mathrm{n}$. 4, 14-15). La formazione delle masse, ${ }^{20}$ e più nello specifico quella delle donne, è oggetto di diversi brani: Amore e capriccio, ovvero le conseguenze di una cattiva educazione (BoRDIGA, LDI, n.6, 21-23), Atti generosi delle donne d'Italia per la causa dell'indipendenza (LDI, n. 14, 54), ${ }^{21}$ Pensieri sull'educazione femminile (ELENA, LDI, n.14, 55-56 e n. 15, 56), Vantaggi della donna istruita (GIACCHIERI, LDI, n.7, 26) sono solo alcuni dei titoli degli articoli volti a sollecitare le giovani donne, future mogli e madri, ad accrescere la propria cultura allo scopo di educare correttamente a propria volta i figli, futuri cittadini. 
L'educazione, del resto, è intesa in questa sede soprattutto come educazione civile e, conseguentemente ad una visione diffusa in Italia a partire dal triennio giacobino (GUERCI 1999), si configura soprattutto come educazione alla storia, tramite la quale gli antichi fasti del Paese vengono rievocati e viene alimentato l'orgoglio nazionale. ${ }^{22}$ Ma qual è il ruolo di queste donne e quale deve essere il limite della loro emancipazione? Il tema è dibattuto nelle pagine del giornale, e si registrano opinioni diversificate: Bardo dei Bardi, ad esempio, ritiene che la donna seppur emancipata debba innanzitutto badare ai figli:

La donna emancipata, a cangion d'esempio, non dovrà già montare come noi la sentinella coll'arme impugnata. Dio la destinò a una guardia egualmente nobile presso la culla dei figli (DE BARDI, LDI, n. 4,13-14)

A giudizio di Bordiga, invece, le donne dovrebbero intervenire in battaglia per salvare la propria patria esattamente come gli uomini:

Ma la donna, benché obliata, fece da sé. Al grido - Abbasso il Borbone! - inalzato in Sicilia, essa pure prese le armi, e combattè contro i sanguinarii soldati, e fece scempio di questi vili strumenti della tirannide. Al grido - Morte all'Austriaco. Innalzato in Milano e ripetuto in tutta la Lombardia, essa pure impugnò il moschetto, e corse a prendere il suo posto alle barricate» (BORDIGA, LDI, n.10, 38-49).

La questione assume una forma più complessa nella rubrica Varietà del numero quarto del giornale, ${ }^{23}$ in cui viene pubblicato un articolo sulla singolare sorte del signor Crobait, impiegato al ministero della guerra e antico ricevitore di dogane a Parigi, che era stato un ammiratissimo funzionario fino alla sua morte, quando si era scoperto che in realtà era una donna. L'articolo non condanna la bugia della signora Crobait, che probabilmente aveva preso il posto di suo marito morto in guerra vivendo così una vita di inganni, ma, al contrario, sottolinea il suo irreprensibile comportamento e il suo essere un «bell'ingegno» ipotizzando che probabilmente aveva sostituito il marito come Tenente anche nelle battaglie di Waterloo e di Moskova. Sembra, in effetti, che queste brevi righe vogliano mostrare le potenzialità delle donne italiane e incitarle se non a imitare la vedova, perlomeno a prendere esempio dalle sue iniziative.

Le «donne in armi» (GUIDI 2000, 571-587) sono personaggi che rientrano spesso nella retorica patriottica e che arricchiscono il catalogo delle "donne 
illustri” di cui si parlerà più approfonditamente in seguito. Si tratta di figure le cui vicende sono rilevanti poiché innescano a partire dal Risorgimento un mutamento nel modo di concepire il genere dettato da una necessità di «manipolazione dei ruoli» (RIALL 2007, 280) che si ritrova spesso negli scritti contestuali (MORI 2011, 99 e BANTI 2000, 193-196). I compilatori vogliono indurre i lettori a chiedersi se la donna debba essere solo educatrice o se possa partecipare anche all'azione bellica, ma i suoi diritti civili, come accade spesso in questi anni, non sono mai discussi (Chemello 2011, 254). I redattori nel complesso dibattito sulla questione si pongono dalla parte di coloro che ritengono che le donne debbano essere, salvo in casi eccezionali, innanzitutto brave mogli e madri di patrioti (CONTI OdORISIO 2010, 47-68).

Tale intento pedagogico viene supportato dalla scelta di inserire nel foglio racconti morali, tra i quali ne spicca uno in particolare, ovvero Un capitolo delle Mie prigioni di Giorgio Pallavicino Trivulzio, preceduto da una breve nota biografica sull'autore (LDI, n.8, 31, n.9, 35-36).24 In questa sede l'articolo²5 assume un valore soprattutto educativo. Il racconto narra l'ultimo smacco che Trivulzio riceve in prigione: l'uomo in confessione affida al parroco tutto quello che ha di più caro, ovvero i suoi denari, e due libri che potrebbero causare pericolose ripercussioni per la sua famiglia qualora fossero scoperti: un romanzo di Cooper e uno di Goethe. Il supervisore, Tomaso Fabri, sequestra tuttavia i volumi e induce il cappellano ad ammettere che appartengono al Trivulzio. Pallavicino è costretto a questo punto ad affrontare un processo e a negare di possedere i libri e il denaro per proteggere la famiglia; la menzogna causa gravissime conseguenze: per spingerlo a rivelare la verità gli austriaci lo riducono alla fame. L'unica consolazione dell'uomo sono le donne che dal carcere femminile oltre il giardino gli rivolgono parole di conforto. Infine Pallavicino confessa, ma quello che qui conta sottolineare è che il racconto è una summa di tutti gli ideali contenuti ne «La donna italiana»; le gravi offese subite dal protagonista, infatti, sono causate da due libri. La cultura assume ancora una volta un valore cruciale, mentre viene alimentato l'odio nei confronti dello straniero oppressore che la ostacola. Le donne, dal canto loro, hanno un merito 
altrettanto importante: sono le uniche che, pietose, assistono alle vicende di Pallavicino e cercano di dargli aiuto. L'inserimento del brano in questa sede ha così una funzione civile nel senso specifico di "civile" in quanto "morale".

Accanto agli scritti di carattere esemplare, ad ogni modo, è presente la cronaca dei moti del 1848 che Bordiga puntualmente riporta in ogni numero. Il Programma iniziale, in cui il direttore esalta Pio IX, non è mai smentito e mette in evidenza una linea politica giobertiana: il Pontefice deve essere a capo dello Stato e i suoi cittadini devono avere fede in lui; tuttavia talvolta il direttore lascia prevalere la sua opinione personale. Quando Pio IX si rifiuta di dichiarare guerra all'Austria, ad esempio, Bordiga manifesta a chiare lettere il suo disaccordo. ${ }^{26} \mathrm{Nel}$ momento in cui Pio IX scende in campo con le sue truppe, coadiuvato da Carlo Alberto, ${ }^{27}$ altro eroe agli occhi dei patrioti, la delusione è però subito dimenticata: «Stringiamoci dunque noi italiani intorno allo stendardo della unità nazionale. L'aureola celeste della religione lo circonda, e lo guida alle vittorie ai trionfi!» (BORDIGA, LDI, n. 4, 14).

Gli articoli sul periodico sono una testimonianza non solo delle oscillazioni del pensiero popolare - e di quello di Bordiga - ma anche della varietà delle posizioni politiche di quegli anni. Sebbene il direttore segua una linea politica giobertiana, infatti, nel giornale sono riportati punti di vista diversi, provenienti anche da Oltralpe. Nel numero quinto si leggono le parole di Lamennais su $I$ nuovi destini d'Italia ${ }^{28}$ seguite da quelle di Mazzini, che esorta i giovani ad arruolarsi alle porte dell'avvento della Repubblica (MAZZINI, LDI, n.16, 62). La presenza delle voci dei due pensatori nel periodico forse non è casuale, in quanto in entrambi, seppure in maniera diversa, «si scorge l'idea di una democrazia quale forma di governo con un'azione pedagogica e con forte senso del dovere e dell'abnegazione» (Guccione 2005, 470), idea che è anche alla base della rivista in esame. I compilatori vogliono in realtà contribuire ad un processo di «disciplinamento eroico» della donna (SolDANI 2007, 217) supportato dal giornale tramite la pubblicazione di rubriche in cui sono narrati i sacrifici compiuti dalle donne di tutta Italia per la patria. Si legga ad esempio il seguente estratto: ${ }^{29}$ 
col cuore commosso, coll'animo acceso dall'entusiasmo, o donne di Roma noi vogliamo farvi nota la grandezza di animo, delle vostre consorelle Bolognesi. Al giungere delle Romane legioni in quella città, ogni cuore avvampò di un fuoco novello. [...] le donne, ben anche, le donne si unirono agli uomini nella impresa di soccorrere la patria. In quei momenti di commozione, fu vista una giovinetta bella quanto povera, la quale non sapendo cosa offrire, si recise i lunghissimi capelli, ne trasse dalla vendita circa 3 scudi, e corse a depositarli sul banco. [...] Donne di Roma! Questi esempi di sublime eroismo v'infiammino il cuore! Emulate, emulate queste magnanime eroine, siate voi pure gli angeli salvatori della patria! Donne di Roma scuotetevi! Colle vostre parole ispirate in ogni petto il più vivo entusiasmo (LDI, n.3, 10).

Il passo vuole addurre come esempio di rettitudine per le donne di tutta Italia, e in particolare per le romane, le imprese delle bolognesi. A questo segue un componimento di Teresa Gnoli3o in cui sono evidenti gli ideali tipicamente risorgimentali: la spinta alla fratellanza, la lotta alle guerre fratricide e il bisogno di costituire uno stato unitario. L'inno, che a livello contenutistico ricorda molto quelli omonimi di Mameli, composto l'anno precedente, di Mercantini o di Berchet, ${ }^{31}$ si conforma ad un «canone risorgimentale» consolidato alla data del

' 48 (BANTI, 2000, 3-55) e, a detta di Raggi,32 diffusosi tra i combattenti tramite fogli volanti a stampa,33 ha un tale successo da diventare introvabile:34

Siam tutti fratelli! Per l'Italo suolo / È sola una speme, un cantico è solo. / Concordia del mondo reina verace / Ci guida alla guerra ci guida alla pace. / Un'era di gioja s'appresta alle genti: / D'ardore possente son piene le menti. / Un grido da segno di giorni novelli: / Siam tutti fratelli! / Il vero trionfi, periscan gl'inganni; / Ai giusti vittoria, infamia ai tiranni; / Finiscan le ambascie, finiscan le pene, / Il peso gittiamo di nostre catene; / Cerchiamo le glorie, sfidiamo le morti; / Vinciamo da grandi, moriamo da forti; / E un grido le imprese d'Italia suggelli: / Siam tutti fratelli! / Già scorre un'amara tristissima etade / Che a nostra ruina stringemmo le spade, / $\mathrm{Ne}$ petti fraterni s'accese la guerra; / Del sangue fraterno si tinse la terra; / Oh giorni di pianto! La vostra memoria / De' giorni presenti non turbi la gloria / Un velo d'orrore tiriamo su quelli: / Siam tutti fratelli! [...].35

\section{Una rete di donne per unire la patria}

Le pagine del periodico offrono alle patriote soprattutto uno spazio in cui confrontarsi sulle proprie esperienza belliche; le donne qui si raccontano e si incitano a vicenda tramite brevi bollettini di guerra, come Alle donne degli stati sardi. Le donne lombarde (LDI, n.8, 30), Le donne bolognesi alle donne toscane 
(LDI, n.12, 47) seguito da Risposta delle donne toscane allindirizzo delle egregie donne bolognesi (LDI, n.13, 51), Le donne pisane alle donne lombarde (LDI, n. 10, 39), o L'Indirizzo delle donne piemontesi alle donne lombarde (LDI, n.4, 16), cronaca del coinvolgimento femminile nelle cinque giornate di Milano. ${ }^{6} \mathrm{La}$ pubblicazione di queste notizie sul foglio non è inusuale, infatti molte delle riviste del periodo danno spazio ad articoli che testimoniano la forte partecipazione delle donne italiane ai moti (SOLDANI 2007, 216-224). Il filo conduttore di tutti i numeri è l'inno alla rivolta, che torna in più occasioni, come ne La crociata contro gli austriaci - appello agli italiani, articolo in cui Bordiga ribadisce il bisogno di scacciare gli austriaci,37 $\mathrm{e}$ in altre forme, come nella Cronaca teatrale settimanale, ${ }^{38}$ in cui talvolta sono pubblicate reazioni forti del pubblico dinanzi a rappresentazioni patriottiche.

I versi delle donne che combattono per i propri ideali sono la parte più peculiare di questo giornale: sin dal primo numero sono pubblicate poesie come L'addio del crociato alla sorella di Virginia Garelli Dalbono (LDI, n.1, 3), seguita nel numero successivo da un'ode di Francesca Cantalamessa rivolta Alla milizia italiana. ${ }^{39} \mathrm{Si}$ tratta di componimenti che hanno in oggetto temi e motivi frequenti nella poesia patriottica, come l'esilio, 40 la lotta allo straniero, in un discorso che come in scritti contestuali si sviluppa «intorno a parole-chiave quali patria, guerra, armi, bandiera, inno» (MORI 2011, 17, 79-106). Nella poesia rivolta $A l$ conte Terenzio Mamiani della Rovere di Elena Montecchi (LDI, n. 4, 45), in particolare l'esilio di Terenzio è preso dalla poetessa a modello per discutere la questione dell'allontanamento degli uomini di ingegno italiani:

e di duri ceppi fremere / a me additava il dolce patrio suolo, / ché in estranie contrade / il fior de' prodi suoi / esular vedea quasi ogni cittade, / e il tuo sovrano ingegno / pur dannato, o Terenzio, a esiglio ingegno.

Tra tutti, ad ogni modo, i più presenti sono i temi della guerra, rivestita di un valore sacro, ${ }^{41}$ e della fratellanza. Ne l'Addio al fratello tenente in cavalleria, crociato italiano di Elvira Giampieri Rossi l'addio tra la poetessa e il suo fratello carnale diventa il simbolo dell'addio tra tutti i soldati d'Italia e le rispettive famiglie: 
Su...fratello! Al campo vola / non guardare al mio pallore! / negli affetti, la parola / non muoviamo per pietà! / Va! Ti chiamano i fratelli della prode Lombardia! / vai fra gli Itali drappelli / Dov'è in armè il patrio amor. / Ma il desio della vittoria / già nello sguardo ti sfavilla! / Dammi un bacio, ed in memoria / questo bacio resti con me. / Nel serrarti al mesto core / premo il simbolo adorato!! / Santa croce del Signore / il fratel riporta a me (LDI, n.4, 15-16).

Da tali estratti risulta evidente quindi che i brani perorano la causa unitaria tramite il ricorso ad un formulario consolidato tipico del «canzoniere risorgimentale» (QUONDAM 2011, X-XX); si legga anche:

E a' fratelli, che in vita restaro, / protendendo i morenti la mano, / disser loro: col sangue italiano / cancellate dal cor la pietà. / Dalla tregua al suonar l'ultim'ora / imbrandite di nuovo le spade, difendete le vostre contrade, / ritogliete le vostre città (Poesia per la resa di Vicenza, inno di Gommi, LDI, n. 13, 50-51).

in cui la retorica patriottica è immediata. Per i compilatori è importante sottolineare che il «vero poeta» - come sostiene Enrico Rinaldi nel presentare i Nuovi canti di Luisa Amalia Paladini - «deve essere nazionale» (LDI, n.10, 38), in cui per «nazionale» si intende qui, sulla base di un comune sentire, ciò che è «rivolto alla collettività» (BANTI 2000, 56-60). Tale assunto si traduce in una serie di versi che spingono esplicitamente alla rivolta, tra questi quelli della suddetta Paladini: «All'armi all'armi! Grida / la magnanima prole di Quirino / all'armi! Dio ci guida! / Italia sorga e vincerà il destino. [...]» (PALADINI, LDI n.10, 38), o quelli di Poesia alle donne italiane in cui predominano l'immagine della bandiera, simbolo dell'antico onore nazionale, e della sorellanza:

Italiane sorelle, ci desti / della patria la voce possente, / della patria già oppressa e morente / or risorta alla speme al valor. [...] noi sorelle, noi tutte Italiane / Sventoliamo la Patria Bandiera / Viva, viva L'Italia, e chi impera / difendendo l'Italico onor.» (LDI, n.6, 24).

Quest'ultimo componimento, firmato da «Una Italiana», offre l'occasione di confrontarsi con una ulteriore pratica diffusa tra le patriote, ossia quella di firmarsi simbolicamente come «donne» o «madri» (MORI 2011, 72).

L'idea che le donne debbano sacrificare i loro affetti personali in nome della causa nazionale, presente in tutto il periodico, si trova infine anche in Patria $e$ libertà di Carlotta Gommi, in cui tornano sia il tema sepolcrale proposto da 
Bordiga in apertura sia la raffigurazione della donna come madre dei futuri cittadini:

[...] O voi madri nel cuore dei figli / della gloria destate il desio / dite loro, che in oggi s'aprio / il sentiero di virtude e d'onor: / [...] che d'Italia fin sacra è la polve, che ogni zolla ricopre la salma / d'un guerriero, che colse la palma / combattendo nel patrio suol (LDI, n.9, 35).

Anche l'immagine della donna come Madre Patria, tra le più evocative e distintive della poesia risorgimentale (BANTI 2000, 5-108), infine è qui frequente nelle sue diverse declinazioni: si leggano in proposito ad esempio Dolce terra materna, illustre affetto di Assunta Pieralli: «Or che ti mostri alfin qual fosti un giorno / Gloriosa d'eroi madre feconda, / Perdona al pianto, che mio ciglio inonda» (PIERALLI, LDI, n. 11, 42), in cui la Madre appare come pietosa e consolatrice, o Le donne italiane agl'italiani redenti di Elisabetta Fiori, in cui l'Italia è raffigurata nella sua abituale iconografia di nutrice che porge il seno nudo, con l'abito lacerato in seguito all'offesa perpetrata dallo straniero (BANTI 2000, 67): «Non udite il lamento, onde v'appella / Italia sanguinosa, e non vedete, / Che a voi lacero mostra il seno ignudo?» (FIORI, LDI, n. 5, 18-19).

\section{Un catalogo parlante}

«La donna italiana», come si è detto, raccoglie profili biografici di donne meritevoli; i racconti delle vite di protagoniste del Risorgimento come Cristina di Belgioioso,42 Maria Giuseppina Guacci Nobile43 e Ermina Prezzolini (Luzi, Ldi, num. 14, 56) sono affiancati a quelli di personaggi del passato, come Cristina da Pizzano,44 definita «un'italiana maestra di liberalismo» (LDI, n. 12, 48),45 Lucrezia Mazzanti (RINALDI, LDI, n. 1,1), ${ }^{6}$ Bianca della Porta (LDI, n. 10 p. 39) e a ricognizioni su Le donne Illustri dalla Roma antica al medioevo (BARBERI, num. 6, 23).

Mentre la vicenda di Erminia è tesa ad esaltarne principalmente le doti artistiche, le altre storie raccontano le vicende di giovani donne incorse nella morte nel tentativo di sfuggire ad uno stupro da parte di un soldato straniero. Anche questo è un motivo topico nella narrativa risorgimentale (BANTI 2000, 
141): la donna che preferisce morire piuttosto che essere violata tiene alto l'onore nazionale. La vicenda di Lucrezia Mazzanti, del resto, è soggetta a diversi riusi nella letteratura coeva, si ritrova, ad esempio, nell'Assedio di Firenze di Guerrazzi, o nel componimento titolato, appunto, Lucrezia Mazzanti, di Isabella Rossi (MORI 2011, 95).

La pubblicazione di scritti e volumi contenenti biografie di donne illustri - dei quali forse la prova più nota è il Prospetto biografico delle donne italiane rinomate in letteratura, di Ginevra Canonici Fachini (FACHINI 1824), che riporta le biografie delle più importanti donne di cultura italiane dal '300 fino all'attualità - nei primi anni dell'Ottocento è frequente (CHEmello 2010, Sinopoli 2010) e, se in alcuni casi ha solo l'obiettivo di enumerare le letterate italiane di maggiore rilievo, in altri si riserva lo scopo di delineare i caratteri di cittadine modello a scopo pedagogico.

Il giornale di Bordiga, tuttavia, non si limita a raccogliere e riferire vicende esemplari, ma lascia alle donne illustri lo spazio per intervenire in prima persona con i loro scritti, dando vita ad un catalogo parlante. Il fatto che «La donna italiana» sia pubblicato a Roma e che ci siano numerosi appelli alle donne romane, inoltre, probabilmente non è casuale, perché esaltare le romane significa esaltare tutte le donne d'Italia in nome dell'antica Roma imperiale. 47 Anche questa associazione non è inusuale: il trattato Delle donne illustri italiane dal XII al XIX secolo (ANONimo 1855), ad esempio, inizia proprio con un appello alle Romane, in cui per romane in realtà si intendono le italiane.

La letteratura delle donne e per le donne diventa quindi in questa sede soprattutto strumento di mobilitazione politica, parte attiva del processo di unificazione nazionale. Il fatto non è straordinario: molta della produzione letteraria in età preunitaria in Italia ha lo scopo di perorare la causa patriottica, 48 e la letteratura femminile non fa eccezione (BALESTRACCI 2015 e CRIVELLI 2012). Anche le forme del discorso sono quelle tipiche del Risorgimento: poesie, prose e racconti morali si affiancano al bollettino della guerra e alla cronaca teatrale. 49

La donna, tuttavia, secondo il direttore deve essere in primo luogo integra e attenta al vero, e di conseguenza questo non risparmia, quando lo ritiene 
necessario e opportuno, critiche anche nei confronti della donna illustre per eccellenza di quegli anni: Cristina di Belgiojoso. Cristina, che nel numero 4, è presentata come un modello da seguire, è oggetto di un'aspra ammonizione nell'ultimo numero del periodico (LDI, n.24, 95-96), in cui il direttore la accusa di avere mosso delle critiche agli italiani senza una opportuna documentazione. Argomento di discussione è il saggio dell'autrice Sull'Italia e sulla Rivoluzione Italiana del $1848^{\circ}$ nel quale si discute delle pessime condizioni dei combattenti italiani al fronte. Bordiga rettifica quanto asserito dalla Belgiojoso e aggiunge che molto spesso si parla male dell'Italia senza avere prove sufficienti. L'intero ultimo numero del periodico, in effetti, consiste in una requisitoria del direttore contro l'armistizio di Salasco e in una esortazione a tornare in battaglia sotto la guida di Carlo Alberto.

Al di là della delusione finale di Bordiga, ad ogni modo, è rilevante il susseguirsi sulla rivista di nomi di autori e autrici di diversa provenienza che testimoniano una attiva rete di patrioti diffusa su scala nazionale. Non bisogna poi tralasciare il fatto che alcuni articoli del giornale, come Pensieri e consigli di celebri donne, Un capitolo delle Mie prigioni di Giorgio Pallavicino Trivulzio e gli Atti generosi delle donne d'Italia per la causa dellindipendenza siano stati pubblicati contestualmente da altri periodici dell'epoca di area milanese, torinese e bolognese. Il fatto che «La donna italiana» contenga questi estratti è segno quindi anche di una facilità nella circolazione della cultura nello Stato Pontificio. ${ }^{1}$

Alla luce di questa analisi, si può affermare, dunque, che la rivista sia testimonianza innanzitutto di una forte volontà dei compilatori di contribuire al processo di unificazione riportando le voci di un popolo letterario femminile riconosciuto e comunemente apprezzato. 


\section{Note}

${ }^{1}$ Cfr. FARINI: «A' 12 marzo il Cardinale Gizzi Segretario di Stato pubblicò un Editto, il quale confermava un altro Editto del 18 agosto 1825 in quella parte che riguardava la censura scientifica, morale e religiosa, ma rispetto alla censura politica istituiva un Consiglio o Magistrato composto di quattro laici ed un ecclesiastico. Ogni cittadino avrebbe facoltà di pubblicare le opinioni ed i giudizi proprii sugli argomenti di amministrazione e di storia contemporanea, purché il facesse in tali termini che né direttamente, né indirettamente tendessero a rendere odiosi gli atti e gli uomini del Governo» (FARINI 1992, 206). La situazione politica e culturale dello Stato Pontificio tra restaurazione e Risorgimento è stata oggetto di diversi studi critici e, negli ultimi anni, in occasione del centocinquantesimo anniversario dell'Unità, di un rinnovato interesse. Per una più approfondita e ampia contestualizzazione cfr. ALFONZETTI - TATTI 2013; BINI 1999; CAFFIERO - DONATO - ROMANO 2005, 171-178; BOUTRY - TRAVAGLINI 1994; LuTTAZZI 2001, LUTTAZZI 2004; MONSAGRATI 2014; TICCHI - LEVILLAIN 2006; TRAVAGLINI 2000, 233-272.

2 Il «Giornale arcadico» nasce nel '18, mentre «L'Album» nel '34, lo stesso anno in cui Jacopo Ferretti fonda «Lo Spigolatore». In queste riviste è evidente l'intento di diffondere le questioni letterarie più attuali. Per fare alcuni esempi si rimanda a L'Italia. Canto IV del Pellegrinaggio di Child Harold, Scritto da Lord Byron, e tradotto da Michele Leoni (ARCADICO 1819, 141-153); Pope, Il riccio rapito tradotto dalla Malvezzi (BETTI 1822, 272-279); Le Bonheur A Elise (ARCADICO 1822, 419-427); Versioni di tre favolette russe di Krylov, (MONTI 1823, 50-56). «Lo Spigolatore» invece riporta una rubrica con i consigli di lettura che riguardano anche volumi stranieri, ad esempio in SPIGOLATORE 1834, 1, è raccomandata la lettura di Salmigondis-Contes de toutes les couleurs, Tome Premier Bruxelles 1833 (che contiene 5 racconti storici di letteratura straniera), mentre il n. 22 (1835) rimanda alle ultime novità edite su «La Biblioteca italiana».

3 Cfr. FARINI: "Alcuni dotti e civili sacerdoti scrivevano il giornale il "Labaro" con generosità d'affetti e temperanza d'opinioni. L'"Epoca”, nata di recente, pareva abbastanza temperata: ma l'uno e l'altro giornale avevano pochi lettori tra il volgo liberale, il quale si nudricava delle pagine del "Contemporaneo"» (FARINI 1992, 253). Per meglio inquadrare la varietà delle posizioni politiche dei periodici romani cfr. MAJOLO MOLINARI 1963, XV-XCL.

4 Cesare Bordiga, direttore dalle idee chiaramente innovative, dopo la fine della Repubblica dirigerà a Firenze, tra il 1851 e il 1864, il «Buon Gusto».

5 Si rimanda in proposito a FRANCHINI 2007, vol. I, 18, in cui si sottolinea che non è possibile classificare rigidamente le riviste femminili in categorie.

${ }^{6}$ Gli scritti femminili di argomento politico sono, soprattutto a partire dal '48, molto diffusi. Si vedano in proposito Vogliamo prender parte agli interessi della patria (CORTESI 1848) tratto dal Proemio al primo numero del «Circolo delle donne italiane» e Caterina FrANCESCHI FERRUCCI (Firenze), Della repubblica in Italia. Considerazioni, Milano, Vallardi, 1848 in PISANO 1994, 336342. Per una approfondita ricognizione sugli scritti femminili di carattere patriottico nel Risorgimento, sia in prosa, sia in versi, si leggano i recenti studi di CHEMELlo 2010 e 2014, MORI 2011.

7 Cfr. CASTRACANE MOMBELli 1998, 10-14 e FARINI 1992, 267-270.

${ }^{8}$ Sul legame tra Sterbini e Angelo Brunetti, detto Ciceuracchio cfr. TREBILIANI 1972.

9 Rossi è a favore della fondazione di uno Stato federalista che dia alla Chiesa la piena autonomia e la possibilità di dichiararsi neutrale rispetto alla guerra in atto. Cfr. FARINI: «Pellegrino Rossi diede opera a formare un Ministero con questi intendimenti: avere soci uomini di opinioni temperate, ma sinceri estimatori e fautori degli ordini liberi; porre ad atto ed esplicare lo Statuto in ogni parte secondo le dottrine e le consuetudini costituzionali; correggere e reprimere ambo i partiti contrarii a quello; cassare privilegii, ristorare l'erario, riordinare l'esercito, stringere lega col Piemonte e colla Toscana, se non si potesse con Napoli; determinare il contingente d'armati, che il Papa darebbe; in guisa che non dovesse versarsi nelle questioni di guerra» (FARINI 1992, 365).

10 Per un approfondimento sul ruolo delle donne a Roma durante la Repubblica romana si cfr. MONSAGRATI 2014, 106-120 e SEVERINI 2011, 99-110.

${ }^{11}$ Da questo momento «La donna italiana: giornale politico letterario» sarà indicata come LDI, i nomi degli autori degli articoli, se presenti, saranno esplicitati. 
12 Teresa Gnoli (1833-1886) ed Elena Montecchi Torti (1814-1868) sono state due note autrici romane. Per un approfondimento sulle loro figure si rimanda a DI CASTRO 2001, vol. 57; FABI MONTANI 1869 e LICAMELI 2013.

13 Per un approfondimento sulle letterate del primo Ottocento si cfr. BELLUCCI 2010 e CORABI 2011, 162-176.

14 Sulla figura di Meucci si cfr. TATTI, 2013, 101-115. Altri collaboratori sono Giuseppe Angelini, Pio Barbieri, Giovanni Boschi, Alessandro Bulgarini, Filippo Raffaeli Marini, Celestino Masetti, Massimiliano Moro, Enrico Rinaldi, Piero Veroli.

15 ENRICO RINALDI, Lucrezia Mazzanti, racconto storico LDI n. 1, 1-2; R. BERLINGHIERI, Cristina Principessa Trivulzi Belgiojoso, LDI, n. 4, 14; PIO BARBERI, Le donne illustri, LDI, n. 6, 23-24; Bianca della Porta, LDI, n. 10, 39; Cristina da Pizzano, LDI, n. 12, 48; L. LuZI, Erminia Prezzolini a Pietroburgo, LDI, n. 14, 56; Maria Giuseppe Guacci Nobile, LDI, n. 5, 17-18; n. 7, 25.

${ }^{16}$ Amore a Capriccio, ovvero le conseguenze della cattiva educazione, LDI, n. 6, 21-23; RAFFAELE Froli, Pietro Torrigiani, LDI, n. 8, 20; n. 9, 33-34; I contrabbandieri, LDI, n. 12, 45-46; n. 13, 4950; Giorgio Pallavicino, Un capitolo delle Mie prigioni, LDI, n. 8, p. 31, n. 9, 35-36.

17 Virginia GaRElli Dalbono, L'Addio del crociato alla sorella, LDI n. 1, 3 (madre patria); Francesca Cantalamessa, Alla milizia italiana, LDi n. 2, 6; Teresa Gnoli, Siam tutti fratelli, LDI, n. 3, 11; ElENA MONTECCHI ToRTI, Ode al conte Terenzio Mamiani della Rovere, LDI, n. 4, 15; ElVIRA GIAMPIERI V. ROSSI, Addio al Fratello, LDI, n. 4, 15-16; ElISABETTA FIORI, Le donne italiane aglitaliani redenti, n. 5, 18-19; UnA Italiana, Poesia alle donne italiane, LDI, n. 6, 24; Pietro COMM. GIACCHIERI, Bastami sol ch'anco a formar la mente, LDI, n. 7, 26; ASSUNTA PIERALLI, Sulla sacra guerra dell'indipendenza italiana, LDI, n. 8, 30; DOMENICO ZAPPI, Al sommo Vincenzo Gioberti, LDI, n. 9, 30; CARLOTTA GOMmI, Patria e libertà, LDI, n. 9, 35; LUISA AMALIA PALADINI, Alla guardia civica, LDI, n. 10, 38; ANGELO MARIA GEVA, Con la voce che chiude ed apre il cielo, LDI, n. 10, 38; ASSUNTA PIERALLI, Dolce terra materna, illustre affetto, LDI, n. 11, 42-43; MARIA GIUSEPPA GUACCI NoBILE, Le donne italiane, LDI, n. 12, 47; CARLOTTA GOMMI, Poesia per la resa di Vicenza, LDI, n. 13, 50-51; GIUSEPPE MONTANELLI, De' tuoi fiori la fragranza, LDI, n. 14, 54; LUISA AMALIA PALAdINI, La bandiera italiana, LDI, n. 15, 58; CESARE BORDIGA, Grido di Guerra, LDI, n. 17, 67; GIACOMO BORGONOVO, Un vaticinio alla Francia, LDI, n. 20, 79; GIUSEPPE MONTANELLI, Io prigioniero ed esule, LDI, n. 22, 87.

${ }^{18}$ La rubrica venne in seguito riportata in «Teatri Arti e Letteratura», vol. 50, (1848), 73.

19 Sull'intento pedagogico de «La donna italiana» si cfr. DE LONGIS 2002, 261-266 e EAD. 2011, 711.

20 CARLETTI, LDI n. 4, 15 «Facciasi insomma tutto quel più che giovar può al intellettuale incremento delle masse, le quali destate come sono a grandi passioni, esigono del pari di venir sussidiate da un corredo di utili ed altrettanto feconde idee nella estensione delle quali trovare di che appagare lo slancio generoso del nazionale sentimento».

${ }^{21}$ La notizia è ripresa da "La Concordia: giornale politico, morale, economico e letterario», 21 luglio 1848, n. 173 e nello specifico è estratta da una lettera di Luigia Romero al direttore del giornale pubblicata nella rubrica Doni all'esercito liberatore.

${ }^{22}$ Gli studi storico-civili in particolare divengono fondamentali per la costituzione degli Italiani e nel Regno di Sardegna fanno già parte, all'altezza del 1848, dei programmi scolastici con scopi dichiaratamente patriottici. In proposito si rimanda ad ASCENZI 2004, 18-22.

23 F., Varietà, LDI, n. 4, 16.

24 Un capitolo delle Mie prigioni di Giorgio Pallavicino, in «Pio IX», I, n.22, 14 maggio 1848, Milano, Ripamonti. Il brano è una prima redazione dell'ultimo capitolo di Spilbergo e Gradisca, il volume di Trivulzio che verrà pubblicato nel ' 56 . Il capitolo porta già lo stesso titolo che ha nella versione definitiva del romanzo, ovvero Gradisca, ed è stato pubblicato nel maggio precedente da un periodico milanese, il «Pio IX. Giornale politico-letterario-artistico dedicato al bravo popolo italiano». Si tratta di uno scritto importante per diverse ragioni. Trivulzio scrisse le sue memorie per tutta la vita (TRIVULZIO 1886) e questo breve estratto è innanzitutto una dimostrazione del fatto che l'autore aveva concepito sin dal ' 48 il suo romanzo (Gradisca è infatti l'ultimo capitolo del volume). Il racconto di prigionia del Pallavicino si pone, anche tramite la scelta del titolo iniziale, in un rapporto di discendenza dalle Mie Prigioni di Pellico e dalle Mémoires d'un prisonnier d'état di Andryane, ma ha lo scopo di rettificare le asserzioni dei due autori, che, a 
detta del Trivulzio «non sono storici» e dunque non hanno saputo esporre la verità (TRIVULZIO 1886, 20). Per ulteriori informazioni sul «Pio IX. Giornale politico-letterario-artistico dedicato al bravo popolo italiano» si rimanda a LUSERONI 2007, 113-115.

25 I romanzi di Pellico e Andryane furono condannati da Giorgio Pallavicino Trivulzio l'uno per l'eccessivo taglio romanzesco e l'altro per l'esaltazione del Confalonieri che, d'altra parte, ne fu infastidito a sua volta. Si cfr. MIRRI 1961.

26 «Ciascuno nutriva la dolce speranza che Pio IX avrebbe finalmente protestato contro le barbarie degli Austriaci benedicendo al tempo istesso una guerra più che ogn'altra giustissima e santa. Ma la speranza universale venne delusa, e le parole del Pontefice risuonarono tremende in ogni animo gettandovi sconforto e dolore» (LDI, n.3, 11).

27 A proposito di Carlo Alberto si legga anche l'immagine eroica con la quale è presentata la reazione di Carlo Alberto alla richiesta di armistizio: «[...] Il magnanimo Carlo Alberto invaso da nobile sdegno, udendoli ciò, strappò quel foglio dei patti, e gridò: Morremo prima sul campo! E fece schierare il valoroso suo esercito in battaglia [...]» in C. BoRDIGA, Roma 5 luglio, Ibidem, n.16, p.61.

28 «Italiani! L'Europa vi guarda: per consumare l'opera che Dio le ha commesso di compier, ha bisogno del vostro concorso e vi conta: voi non tradirete la sua aspettativa!» (LDI, n. 5, 18).

29 Altri inviti alle donne di Roma sono presenti nel periodico cfr. BORDIGA, LDI, n. 14, 53.

${ }^{30}$ L’inno è preceduto da una introduzione di Oreste Raggi che recita «[...] se Tireo, se Riga tanto poterono, quegli sopra gli antichi, questi sopra i moderni Greci loro cittadini, nello infiammarli coi loro canti alla guerra e alla libertà, quanto non potranno in noi Italiani i versi non dico di un nuovo Tireo, ma delle nostre gentili donne e agli stessi magnanimi sentimenti poetando incitando i concittadini, come una Ferrucci, una Guacci, una Paladini, una Montecchi, una Cantalamessa, una Taddei, una Gnoli e tante altre loro pari? Ben converrebbe che questi versi fossero messi in musica da' valenti maestri, e cantati in mezzo alle italiche schiere quale entusiasmo non desterebbero al combattimento, alla vittoria?» (RAGGI, LDI, n.3, 10-11). L'inno ha grande successo e nel numero quarto de "La donna italiana» Bordiga in un Annunzio tiene a specificare che le parole di Teresa, pubblicate nel numero precedente, non solo saranno musicate, ma diventeranno voce delle milizie italiane: «nel passato numero della Donna Italiana veniva manifestato il desiderio di veder posto in musica l'Inno dell'egregia Teresina Gnoli in esso inserito. Amiamo adesso annunziare che questo voto è stato già appagato, e che il dotto maestro Genoves ha adattato una bella musica a questa ispirata poesia, la quale verrà quanto prima eseguita dai concerti delle nostre milizie» (BORDIGA, LDI, n. 4, 16).

${ }^{31}$ Si veda ad esempio Su! Figli d'Italia di Berchet in cui l'autore, come Teresa Gnoli, sprona gli italiani a gridare all'unisono: «dall'Alpi allo Stretto fratelli siam tutti!».

32 Per un approfondimento sulla figura di Oreste Raggi, studioso di archeologia e storico cfr. PALANDRANI 2016.

33 Una copia di uno di questi fogli è oggi conservata presso l'Archivio Gnoli in Tommaso GNOLI, B14, vol. IX. L'inno è preceduto da una breve premessa degli editori in cui si legge «[...] Evviva voi, evviva l'Italia! Ma non per ciò avrete discaro portarvi in questo inno, che intendiamo offrirvi in ricordo di una così valente nostra concittadina, quale è la giovinetta signora Teresa Gnoli, e le nostre donne ormai, deposta ogni debolezza d'animo che farebbe onta al nome d'Italia e di Roma, devono anzi collo esempio della giovine poetessa incitare alle armi i fratelli, i figli, gli sposi, siccome usavano le antiche Spartane imponendo loro o di tornare vincitori collo scudo, o morti su questo [...]».

34 RAGGI, LDI, n. 3, 11: «[...] pure è sì universalmente ricercata non potendosene oggimai trovare più una copia per qualunque prezzo».

35 Teresa GNOLI, Siam tutti fratelli, Ibidem, n. 3, p.11.

${ }^{36}$ Ivi: «[...] Il vostro coraggio è meraviglia, non solo all'Italia, ma all'Europa! Le vostre gesta pareggiano le Italiane alle Greche, alle Polacche, alle Iberiche. [...] voi insegnaste quanto possa la donna, che sente la propria dignità ad ha fede nella sua missione. Onore eterno al valore delle Milanesi. [...] porgiamoci la mano di amorose sorelle, e siano indissolubili i nostri legami d'affetto, come indivisibili sono e saranno sempre i bisogni e gl'interessi della patria come l'Italia». 
37 BORDIGA, LDI, n. 2, 5: «[...] Dall'Alpe alle sicule spiagge una voce sola rimbomba: Guerra! guerra tremenda! Fuori d'Italia i nostri oppressori! È questa la voce di un popolo che si è sentito in grande ed è sorto a frangere i ceppi col furore di ruinoso torrente che avanti a se tutto schianta e devasta!».

${ }_{38}^{8}$ Esemplare è il racconto dell'esaltazione generata dalla rappresentazione dell'Ariberto da Porta romana, ovvero la cacciata dei tedeschi dallo lombardia, episodio della trilogia di Napoleone Giotti: «Il valente attore brillante Costantino Venturoli [...] declamò un inno lirico con tal forza, con tanto entusiasmo, che trasfuse il suo proprio fuoco nell'uditorio. Quando egli proruppe: Via d'Italia lo straniero, /nessun patto coll'Impero! Tutto il pubblico si levò in massa ripetendo sonoramente: - Via! Via! Nessun patto! - e fece tale un frastuono da porre precipitosamente in fuga tutti i retrogradi (altro termine tecnico) che si fossero trovati in teatro. Di quest'inno il primo giorno si volle la replica» (LDI, n. 13, 52).

39 [...] V' unite o fratelli - versate quel sangue / Per cui dell'Italia - la sorte pur langue / I pochi fratelli - che abborran la madre / Si tolgan col ferro - dell'Itale squadre, / col ferro che ai vili terrore sol dà [...] (LDI, n.2, 6)

40 Per una ricognizione sul tema dell'esilio si rimanda a TATTI 1999; 2007 , vol. 1, 743-749 e a FINELLI 2010, 121-136.

${ }^{41}$ Si vedano Poesia sulla sacra guerra dellindipendenza italiana, canzone di Assunta Pieralli, LDI n.18; In morte del barone pompeo Danzetta di Perugia (capitano aiutante meggiore nelle legioni romane, caduto nella battaglia di Cornuda, il dì 9 maggio 1848) ode di Assunta Pieralli, Ibidem, n. 11, 42-43.

42 R. BERLINGHIERI, Cristina, Principessa Trivulzio di Belgioioso, LDI, n. 4, 14.

43 Cesare Bordiga, Maria Giuseppa Guacci Nobile, ibid., num.5, 17; n. 7, 25.

44 Per un approfondimento sulla figura di Cristina da Pizzano si cfr. FACHINI 1824, 81; SINOPOLI 2010, 31-44.

45 Per quanto riguarda la figura di Cristina da Pizzano si cfr. CANONICI FACHINI 1824, 81.

${ }^{46}$ Sulla funzione esemplare di Lucrezia Mazzanti nel Risorgimento si cfr. BALESTRACCI 2015, 6465; BANTI, 2000, 100-101.

$47 \mathrm{Si}$ veda la canzone rivolta a Le donne italiane di Maria Giuseppina Guacci Nobile in cui la grandezza di Roma, regina d'Italia, e delle romane, è indiscussa: «Qual celeste camena / mi canterà di voi Romane acerbe [...] oh! Riposate in pace, e la serena / Fronte celate negl'infranti avelli, / né più tardi mai cura del Tebro! / [...] Ah d'Italia regina / aure sacre e feconde ! oh vivo sole / che di gigli inghirlandi e di viole / e l'una e l'altra splendida marina! [...]» (LDI, n.12, 47).

${ }^{48}$ Sul valore della politica nella letteratura del Risorgimento si sono espressi in molti, si rimanda in particolare al recente E. CAPUZZO, A. CASU, A. SABATINI 2013 nel quale si affrontano anche questioni riguardanti il caso specifico di Roma (cfr. TEODONIO, Ibid., 119-133).

49 Per quanto riguarda l'incidenza delle cronache teatrali e delle riviste teatrali nella Roma dell'Ottocento si rimanda a O. MAJOLO MOLINARI 1963, LXXIX-LXXX e a MACCHIONE 2008, 177195 .

5o Revue des Deux Mondes, 15 sett. 1848.

${ }^{51}$ Studi recenti hanno evidenziato la presenza di una buona circolazione della cultura a Roma a partire dalla fine del Settecento per poi proseguire nel periodo risorgimentale, in proposito si rimanda in particolare a CAFFIERO - DONATO - ROMANO 2005 e a ALFONZETTI-TATTI 2013. 
Bibliografia

ANONIMO 1855

AnONImo, Delle donne illustri italiane dal XII al XIX secolo, Roma, fratelli Pallotta Tipografi in Piazza Colonna, 1855 .

ARCADICO 1819

L'Italia. Canto IV del Pellegrinaggio di Child Harold, Scritto da Lord Byron, e tradotto da Michele Leoni, in «Giornale arcadico di scienze lettere ed arti», I, (1819), pp. 141-153.

ASCENZI 2004

Anna ASCENZI, Tra educazione etico-civile e costituzione dell'identità nazionale, Milano, Vita e Pensiero, 2004, pp. 18-22.

BALESTRACCI 2015

Duccio BALESTRACCI, Medioevo e Risorgimento. Linnvenzione dell'identità italiana nell'Ottocento, Bologna, Il Mulino, 2015.

BANTI 2000

Alberto Mario BANTI, La nazione del Risorgimento, Torino, Einaudi, 2000.

BELLUCCI 2010

Novella BELLUCCI (a cura di), Per un archivio delle scritture femminili del primo Ottocento italiano in Dimensioni e problemi della ricerca storica: rivista del Dipartimento di studi storici dal medioevo all'età contemporanea dell'Università "La Sapienza" di Roma, Roma, Franco Angeli, 2010.

BETTI 1822

Salvatore BETTI, Pope, Il riccio rapito tradotto dalla Malvezzi, «Giornale arcadico di scienze lettere ed arti», XIV, (1822), pp. 272-279.

BERTOLO 2011

Bruna BERTOLO, Donne del Risorgimento. Le eroine invisibili dell'Unità d'Italia, Torino, Ananke, 2011.

BOUTRY - TRAVAGLINI, 1994

Philippe Boutry, Carlo M. Travaglini, Roma tra fine Settecento e inizio Ottocento in «Roma moderna e contemporanea», 1, 1994.

BRIGANTI-CATTARULLA-D'INTINO 1990

Alessandra BRIGANTI, Camillia CATTARUlLA, Franco D'InTINo, I periodici Letterari dell'Ottocento, Milano, Franco Angeli, 1990.

CAFFIERO - DONATO - ROMANO 2005

Marina CAFFIERO, Maria Pia DONATO, Antonella RomANO, De la catholicité posttridentine a la Republique romaine, in Naples, Rome, Florence. Une histoire comparée des milieux intellectuels italiens (XVIIe-XVIIIe siècles), a c. di J. BOUTIER, B. MARIN E A. RomANO, Roma, École française de Rome, 2005, pp. 171-178. 
CAPUZZO-CASU-SABATINI 2013

CAPuzzo, A. CASU, A. SABATINI (a cura di), Pensiero politico e letteratura del Risorgimento, Rubbettino, Soveria Mannelli, 2013.

CORABI 2011

Gilda CORABI, Scrittrici dell'Ottocento in Atlante della letteratura italiana, a c. di Sergio Luzatto e Gabriele Pedullà, vol. III, Torino, Einaudi, 2011, pp. 162-176.

CASTRACANE MOMBELLI 1998

Mirella CASTRACANE MOMBELLI, Governo, legislazione, amministrazione centrale e locale dello Stato Romano tra il 1860 e il 1860 in Fiorella Bartoccini, Donatella Strangio, Lo stato del Lazio 1860-187o, Roma, Istituto nazionale di studi romani, 1998, pp. 10-14.

CHEMELLO 2010

Adriana Chemello, Fuori dai repertori. Donne sulla scena letteraria ottocentesca in «Dimensioni e problemi della ricerca storica», 1/2010, pp. 45-60.

CHEMELLO 2014

Adriana CHEMELlO, "Nel glorioso avvenire della mia patria". Frauen und Schriftstellerinnen während der Einigung Italiens in 150 Jahre Italien, Themen Wege, offene Fragen, a c. di Florika Griessner e Adriana Vignazia, Wien, Praesens Verlag, 2014, pp. 247-269.

CONTI ODORISIO 2010

Ginevra CONTI ODORISIO, La questione femminile nell'Ottocento e Cristina di Belgiojoso in Cristina di Belgiojoso. Politica e cultura nell'Europa dell'Ottocento, a c. di Ginevra Conti Odorisio, Cristina Giorcelli, Giuseppe Monsagrati, Loffredo Editore, Napoli, 2010, pp. 47-68.

CRIVELLI 2012

Tatiana CRIVELLI, «Deh, non opinate, o Signora, così spregevolmente di noi»: l'Italia illustrata dalle italiane, in altrelettere», <http://www.altrelettere.uzh.ch/article/view/al_uzh-1/305>, rivista online diretta da Tatiana Crivelli, Romanisches Seminar, Università di Zurigo (16.3.2018).

DE LONGIS 2002

Rosanna DE LONGIS, «La donna italiana». Un giornale del 1848 in Genesis, I/1, 2002, pp. 261-266.

DE LONGIS 2011

Rosanna DE LONGIS, Paola GIOIA, Libere e generose sorelle: La donna italiana, 1848, Roma, Biblink, 2011, pp. 7-11.

DELLA PERUTA 2011

Franco Della Peruta, Il giornalismo italiano del Risorgimento dal '47 all'Unità, Milano, Franco Angeli, 2011, pp. 20-23.

DI CASTRO 2011

Raffaella Di CASTRO, Teresa Gnoli, in DBI, Volume 57 (2001). 
DONI et al. 2011

Elena DONI et al., Donne del Risorgimento, Bologna, Il Mulino, 2011.

FABI MONTANI 1869

Francesco FABI MonTANI, Elogio storico di Elena Montecchi Torti, Roma 1869.

FACHINI 1824

Ginevra CANONICI FACHINI, Prospetto biografico delle donne italiane rinomate in letteratura, Venezia, Alvisopoli, 1824.

FARINI 1992

Luigi Carlo FARINI, Antonio PATUELli (a cura di), Lo Stato Romano dall'anno 1815 al 1850, Presidenza del Consiglio dei Ministri-Dipartimento per l'informazione e l'editoria, Istituto Poligrafico e Zecca dello Stato, 1992 [1. ed. Le Monnier, 1853].

FINELLI 2010

Pietro FINELLI (a cura di), L'Italia fuori L'Italia in Nel nome dell'Italia. Il Risorgimento nelle testimonianze, nei documenti e nelle immagini, Roma-Bari, Laterza, 2010, pp. 121136.

FRANCHINI 2007

Silvia Franchini, Introduzione in Silvia Franchini, Monica Pacini, Simonetta Soldani, Giornali di donne in Toscana. Un catalogo, molte storie (1770-1945), Leo S. Olschki Editore, Firenze, 2007, vol. I, p. 18.

GIOIA-ZUCCONI 2013

Paola GioIA, Antonietta Angelica ZUCCONI, I periodici della Repubblica romana nella Biblioteca di Storia moderna e contemporanea di Roma in La Repubblica romana del 1849 la storia il teatro la letteratura, a c. di Beatrice ALFONZETTI e Silvia TATTI, Roma, Bulzoni, 2013, pp. 151-164.

GUCCIONE 2005

Eugenio GuICCIONE, Mazzini e Lamennais tra analogie e divergenze in Mazzini e gli scrittori politici europei (1837-1857) a c. di Salvo Mastellone, t.2, Firenze, Centro Editoriale Toscano, 2005, pp. 461-489.

GUERCI 1999

Luciano GUERCI, Istruire nelle verità repubblicane. Letteratura politica per il popolo nell'Italia in rivoluzione (1796-1799), Bologna, il Mulino, 1999.

GUIDI 2000

Laura GUIDI, Patriottismo femminile e travestimenti sulla scena risorgimentale, in «Studi Storici», XL (2000), num. 2, pp. 571-587.

L., ARCADICO 1822

L., Le Bonheur A Elise, "Giornale arcadico di scienze lettere ed arti», XII, (1822), pp. 419-427. 
LDI 1848

«La donna italiana: giornale politico letterario», Tip. delle Scienze, Dir. Cesare Bordiga, 22 aprile 1848-11 novembre 1848. Settimanale.

LICAMELI 2013

Chiara LiCAMELI, Poetesse a Roma nel Risorgimento, tesi di laurea non pubblicata, a. a. 2012/2013, relatrici Silvia Tatti e Novella Bellucci.

LUSERONI 2007

Giovanni LUSERONI, Giuseppe Mazzini e i democratici del quarantotto lombardo, Roma, Gangemi, 2007, pp.113-115.

MACCHIONE 2008

Daniela MACCHIONE, «Ne' latifondi delle lettere, e delle arti belle» sotto l'occhio vigile della censura. Storia dell'«Eptacordo» in «Fonti musicali italiane», 13/2008, Roma, CIDIM, 2008, pp. 177-195.

MAJOLO MOLINARI 1963

Olga MAJOlO MOLINARI, La stampa periodica romana dell'Ottocento, Roma, Istituto di studi romani, 1963.

MEROLLA 1996

Riccardo MERolla, Lo Stato della Chiesa in Letteratura Italiana. Storia e geografia, diretta da Asor Rosa, vol. II, Milano, Einaudi, 1996.

MIRRI 1961

Mario MIRRI, Andryane, Alexandre-Philippe, Dizionario Biografico degli Italiani Volume 3 (1961).

MONSAGRATI 2014

Giuseppe Monsagrati, Roma senza il Papa. La Repubblica romana del 1849, Bari, Laterza, 2014, pp. 11-27.

MORI 2011

Maria Teresa MORI, Figlie d'Italia: poetesse e patriote nel Risorgimento (1821-1861), Roma, Carocci, 2011.

MONTI 1823

Vincenzo MonTI, Versioni di tre favolette russe di Krylov, in «Giornale arcadico di scienze lettere ed arti», XVII, (1823), pp. 50-56.

PALANDRIANI 2016

Claudio PALANDRANI, Vita e Opere di Oreste Raggi. Ritratto di un carrarese illustre attraverso un compendio antologico dei suoi scritti, Tipografia Apua Service, Massa, 2016.

PISANO 1994

Laura PISANO, Giornalismo politico delle donne italiane delle Repubbliche giacobine al Risorgimento (1796-1860) in Laura PISANO, Christiane VEAUVY, Parole inascoltate: le 
donne e la costruzione dello stato-nazione in Italia e in Francia 1789-1860: testi e documenti, Roma, Editori Riuniti, 1994, pp. 9-63.

QUONDAM 2011

Amedeo QUONDAM, Risorgimento a Memoria, Pomezia, Donzelli Editore, 2011.

RIALL 2007

Lucy RIALL, Eroi maschili, virilità e forme della guerra in in Storia d'Italia. Annali 22. Il Risorgimento, a c. di Alberto Mario Banti, Paul Ginsborg, Einaudi, 2007, pp. 277-285.

SEVERINI 2011

Marco SEVERINI, La Repubblica romana del 1849, Venezia, Marsilio, 2011, pp. 99-110.

SILVAGNI 1971

David Silvagni, Lucio FELICI (a cura di), La corte pontificia e la società romana nei secoli 18 e 19, Roma, Biblioteca di storia patria, 1971 [1. ed. Forzani 1883-1885].

SINOPOLI 2010

Franca SINOPOLI, Da Corinne alle "altre": per un confronto tra Lady Morgan e Ginevra Canonici Fachini in «Dimensioni e problemi della ricerca Storica», XXII, 1, 2010, pp. 3144 .

SOLDANI 2004

Simonetta SOLDANI, Donne educanti, donne da educare. Un profilo della stampa femminile toscana (1770-1945) in Donne e giornalismo. Percorsi e presenze di una storia di genere a c. di Silvia Franchini e Simonetta Soldani, Milano, Franco Angeli, 2004, p. 309.

SOLDANI, 2007

Simonetta Soldani, Il Risorgimento delle donne in Storia d'Italia. Annali 22. Il Risorgimento, a c. di Alberto Mario Banti, Paul Ginsborg, Einaudi, 2007, pp. 183-224.

SPIGOLATORE 1834

«Lo Spigolatore. Giornale di scienze, lettere, costumi, teatri, aneddoti, bibliografia, necrologia», Roma, 1834-1836.

TATTI 1999

Silvia TATTI, Le tempeste della vita: la letteratura degli esuli italiani in Francia nel 1799, Paris, H. Champion, 1999.

TATTI 2007

Silvia TATTI, Esilio in Ceserani, M. Domenichelli, P. Fasano (a c. di), Il grande dizionario enciclopedico dei temi letterari, Torino, UTET, 2007, vol. 1, pp. 743-749.

TATTI 2013

Silvia TATTI, "A tutti gl'Italiani». Letteratura, melodramma e politica da Pio IX alla Repubblica in La Repubblica romana del 1849 la storia il teatro la letteratura, a c. di Beatrice Alfonzetti e Silvia Tatti, Roma, Bulzoni, 2013, pp. 101-115. 
TREBILIANI, 1972

Maria Luisa TREBILIANI, BRUNETTI, Angelo, detto Ciceruacchio in Dizionario Biografico degli Italiani, vol. 14, 1972.

TRIVULZIO 1886

Giorgio Pallavicino Trivulzio, Memorie di Giorgio Pallavicino pubblicate per cura della moglie, Torino, Roux Frassati e C., 1886.

TICCHI - LEVILLAIN 2006

Jean Marc TICCHI, Philippe LEVILLAIN (a c. di), Le Pontificait de Leon XIII: renaissances du Saint-Siège?, Roma, Ecole francaise de Roma, 2006.

TRAVAGLINI 2000

Carlo Maria Travaglini, Aspetti della modernizzazione economica tra fine Settecento e inizi Ottocento. La politica fiscale in Roma, Roma negli anni di influenza e dominio francese, in Philippe Boutry, Francesco Pitocco, Carlo Maria Travaglini, Napoli, ESI, 2000, pp. 233-272. 